\title{
Cervical lymph node enlargement as the initial manifestation of rectal cancer
}

\author{
Tong-Hui Xie ${ }^{1}$, Peng Su ${ }^{2}$, Jian-Guo Hong ${ }^{1 *}$ (1) and Hui Zhang ${ }^{2^{*}}$
}

\begin{abstract}
Background: Colorectal cancer is a very common malignant tumor worldwide. The clinical manifestations of advanced colorectal cancer include the changes in bowel habits, hematochezia, diarrhea, local abdominal pain and other symptoms. However, the colorectal cancer with an initial symptom of cervical lymph node enlargement is extremely rare. In this article, we report a case of rectal cancer presenting with cervical lymph nodes enlargement as the initial symptom.

Case presentation: A 57-year-old woman was admitted to our hospital for cervical lymph node enlargement which was accidentally detected during physical examination. Computed tomography scan revealed multiple enlarged lymph nodes in the neck. Cervical ultrasound showed normal thyroid gland and multiple left supraclavicular lymph nodes enlargement. The patient underwent lymph nodes biopsy and pathologic results showed metastatic adenocarcinoma. The subsequent lower gastrointestinal endoscopy revealed a mucosal bulge lesion located at rectus and biopsy revealed adenocarcinoma. The patient underwent rectal cancer resection. She is alive with no evidence of recurrence or new tumors 2 years after surgery.

Conclusions: Cervical lymph node metastasis is a rare metastatic way in colorectal cancer. This is the first case of rectal cancer presenting with cervical lymph nodes metastases as the initial symptom. Surgical resection combined with postoperative chemotherapy improved long-term prognosis of the patient. This rare metastatic way of rectal cancer should be paid attention for clinicians.
\end{abstract}

Keywords: Colorectal cancer, Lymph node metastasis, Surgery

\section{Background}

Colorectal cancer is the third most common malignant tumor and the second leading cause of cancer-related death in the world, with more than 1.4 million new cases and 0.8 million deaths each year [1]. Lymph node metastasis in patients with colorectal cancer is common, indicating that the tumor is at least in the middle stage [2]. In general, the lymph nodes near the primary site are the most common metastatic site for rectal cancer [3]. In this article, we report a case of rectal cancer presenting with

\footnotetext{
*Correspondence: hjianguo123@126.com; zhanghui3721@126.com ${ }^{1}$ Department of General Surgery, Qilu Hospital of Shandong University, 107 West Wen Hua Road, Jinan 250012, Shandong, China

2 Department of Pathology, Qilu Hospital of Shandong University, 107 West Wen Hua Road, Jinan 250012, Shandong, China
}

cervical lymph nodes enlargement as the initial symptom. This patient had no other symptoms and the skip metastasis was the main form of metastasis for this case.

\section{Case presentation}

A 57-year-old woman was admitted to our hospital for a cervical mass which was incidentally detected during physical examination. She presented no abdominal pain, abdominal distension or gastrointestinal bleeding. Physical examination revealed multiple cervical masses with the maximum diameter of $3 \mathrm{~cm}$. The masses were hard and presented poor mobility.

Laboratory examination showed elevated levels of CEA (15.59 ng/ml, normal range, 0-5), CA19-9 (42.78 ng/ml, normal range $0-37)$ and CA242 $(22.50 \mathrm{U} / \mathrm{ml}$, normal 
range 0-20). Serum levels of CA125, CA15-3 and AFP were within the normal range. The complete blood count, thyroid function and liver function were normal. Cervical ultrasound showed normal thyroid gland and multiple left supraclavicular lymph nodes enlargement with the maximum diameter of $3.3 \mathrm{~cm}$ (Fig. 1a). The patient underwent chest computed tomography (CT) and whole gastrointestinal tract barium X-ray radiography. The CT scan revealed multiple enlarged lymph nodes in the bilateral submandibular region, bilateral carotid sheath and intermuscular space of the cervical root (Fig. 1b, c). Whole gastrointestinal tract barium X-ray radiography revealed no obvious lesion (Fig. 1d, e).

The patient underwent lymph nodes biopsy. Intraoperative frozen section analysis showed metastatic adenocarcinoma cells with marked atypia arranged in nests and cribriform pattern within the lymph nodes. Necrosis and scattered calcification were present within tumor foci (Fig. 2a, b). Based on the histological morphology, the metastatic adenocarcinoma most likely arose from breast or gastrointestinal tract. Given that a mammary nodule was detected on palpation, resection of the mammary nodule was performed during this operation. However, intraoperative frozen section analysis showed mammary gland hyperplasia. After the operation, immunohistochemical examination of the cervical lymph nodes biopsy was performed and showed that the tumor was positive for GCDFP (Fig. 2c), CK19 (Fig. 2d), CK20 (Fig. 2e) and CDX-2 (Fig. 2f), but was negative for actoglobulin, ER, PR and TTF-1.

After the operation, the patient underwent upper gastrointestinal endoscopy but no esophageal or gastric tumors were detected. In order to identify the primary tumor location, pelvic enhanced CT scan was performed and it revealed uneven thickening and enhancement of the rectal wall. The subsequent lower gastrointestinal endoscopy revealed a mucosal bulge lesion located at rectus (Fig. 1f) and biopsy revealed rectal adenocarcinoma.

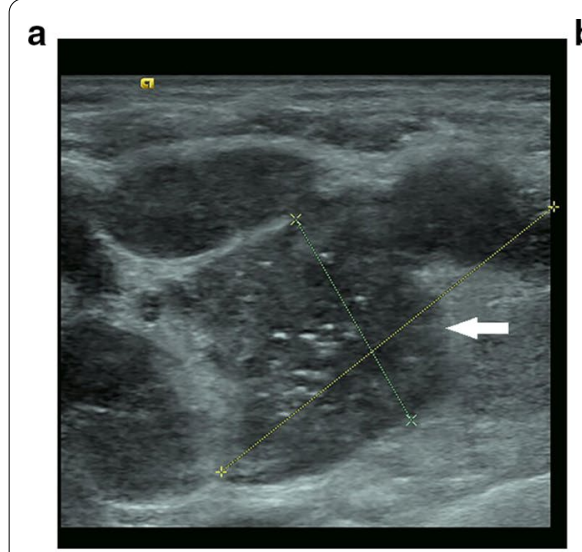

d
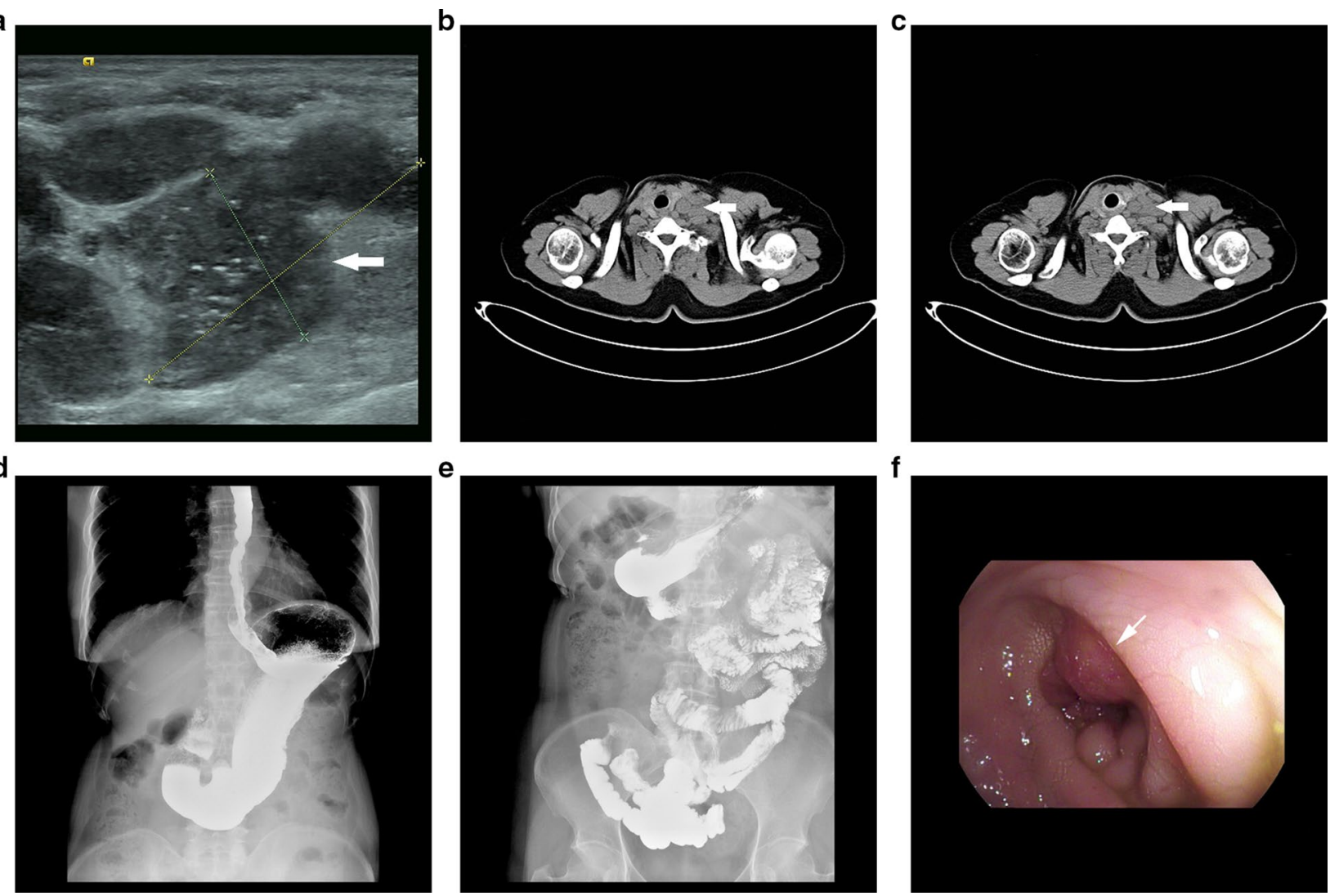

f

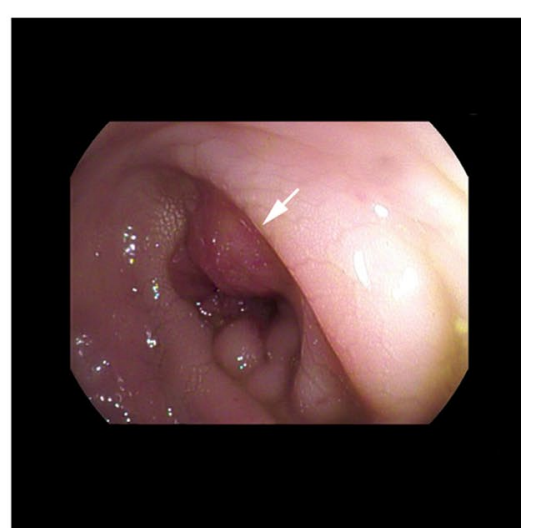

Fig. 1 a (Cervical ultrasound) showed multiple hypoechoic nodules in the left neck and left supraclavicular fossa with clear boundaries, irregular morphology, multiple dotted strong echoes and rich blood flow signals. b, c (Chest computed tomography) showed multiple enlarged lymph nodes in the bilateral submandibular region, bilateral carotid sheath and intermuscular space of the cervical root. $\mathbf{d}$ (Whole gastrointestinal tract barium X-ray radiography) showed whole gastrointestinal tract has no sign of obstruction. e, $\mathbf{f}$ (Lower gastrointestinal endoscopy) showed irregularly elevated rectal mucosa with congestion, erosion and easy bleeding. The lesion caused rectal narrowing and the endoscopy was not able to pass through 


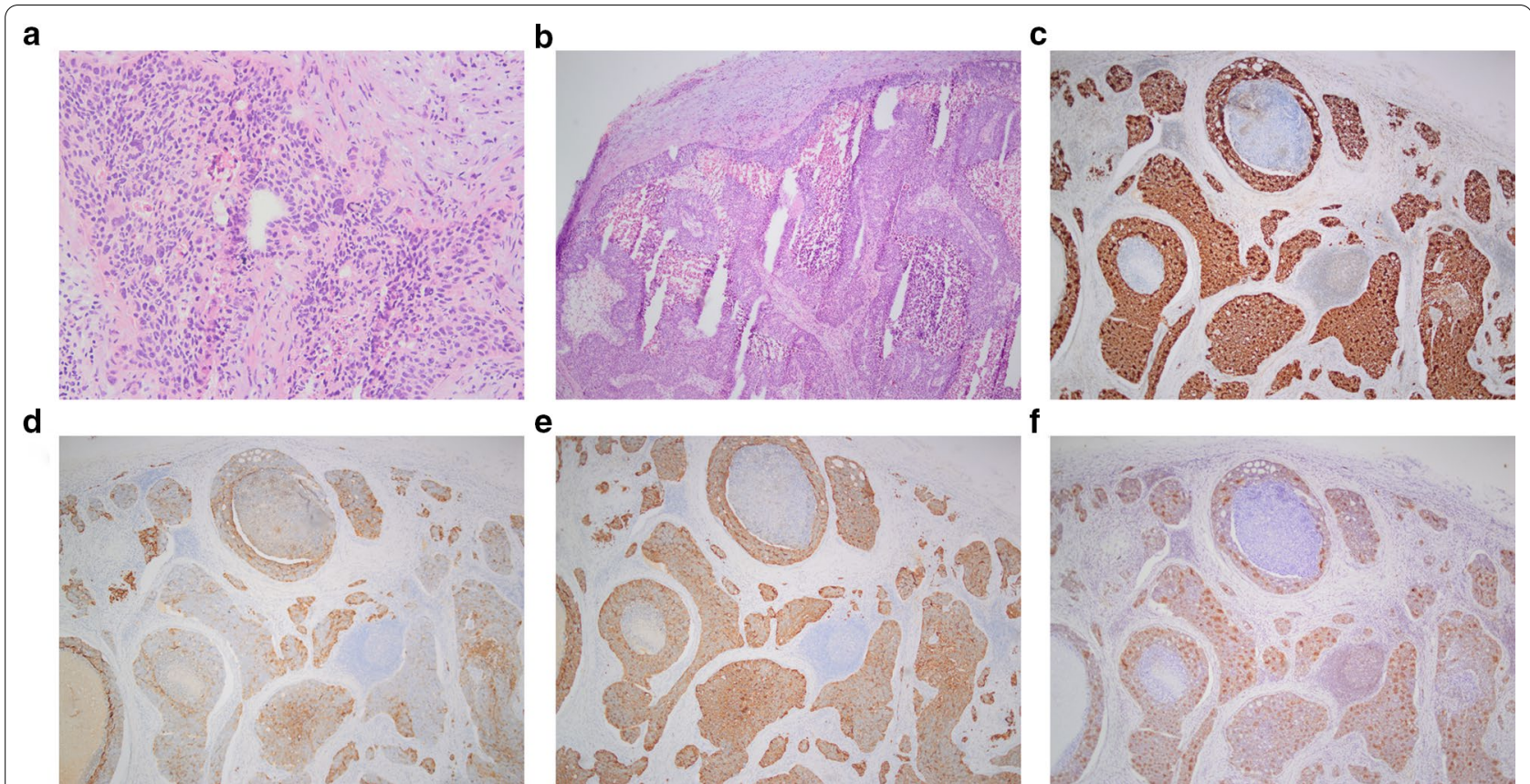

Fig. 2 showed the pathologic results of the cervical lymph nodes biopsy. a (HE staining, $\times 40)$ and $\mathbf{b}(H E$ staining, $\times 200)$ showed metastatic poorly differentiated adenocarcinoma was found in lymph nodes with marked atypia arranged in nests and cribriform pattern. Necrosis and scattered calcification were present in the center of the carcinoma. The tumor cells are heterogeneous with pathological mitotic figures. Based on the histological morphology, the adenocarcinoma most likely arose from breast or gastrointestinal tract. Immunohistochemical examination showed that the neck mass was positive for GCDFP $(\mathbf{c}, \times 40)$, CK19 $(\mathbf{d}, \times 40)$, CK20 $(\mathbf{e}, \times 40)$ and CDX-2 $(\mathbf{f}, \times 40)$

The patient underwent rectal cancer resection. During the operation, we found that the tumor was located at rectum above the peritoneal reflexion. The tumor was hard and the size was about $3 \times 4 \mathrm{~cm}$. The rectal tumor penetrated rectal serosa and invaded the surrounding adipose tissue. Metastatic adenocarcinoma was detected within peri-intestinal lymph nodes. Based on intraoperative exploration and previous medical history, rectal cancer resection was performed and the patient recovered uneventfully. Eventually, based on the patient's postoperative pathologic results, the tumor was diagnosed at stage IV A (T3N2bM1a). The patient recovered uneventfully and no evidence of recurrence or new tumors was found during 2 years follow-up.

\section{Discussion and conclusions}

Colorectal cancer is the third most common malignant tumor and the second leading cause of cancer death worldwide [4]. Direct invasion, hematogenous spread, lymphatic spread and implantation metastasis are the principal ways for metastasis in colorectal cancer. Although most malignant lymphadenopathy in the neck represent lymphomas or metastases from head and neck primary tumors, occasionally, metastatic disease from remote sites presents as cervical lymphadenopathy with or without an obvious primary tumor [5]. The case of rectal cancer reported in this article presented enlarged cervical lymph nodes as the initial symptom without any signs or symptoms of primary organ involvement is extremely rare.

Gastrointestinal tumor metastases in the left supraclavicular lymph node, also known as the Virchow lymph node, is often the sign of advanced malignant disease (stage IV). In 1848, the German pathologist Rudolf Virchow first described it as an enlarged gland associated with gastric cancer. Virchow's node has come to be known as a signal node, signaling the presence of an underlying cancer form a primary lesion in the upper abdomen [6]. The most common digestive tract tumor with supraclavicular lymph node metastasis is gastric cancer. Cervical lymph node metastasis is an extremely rare metastatic way for colorectal cancer. The most common metastatic sites for colorectal cancer were regional lymph nodes and liver. Other sites include lung, peritoneum, ovary, central nervous system, bone, kidney and uterus. Adrenal gland, hilar lymph node, skin and muscle are the extremely rare metastatic sites for colorectal cancer [7].

At present, the mechanism of distant non-regional lymph nodes metastases in patients with colorectal cancer remains unclear. According to previous reports [7], skip micrometastasis between regional lymph nodes can 
be seen in $18 \%$ of cases. This case we reported would contribute to the interpretation of the mechanism of skip metastasis. For postoperative pathology, CDX-2 and CK20 are the most common immunohistochemical type in colorectal cancer [8]. CDX-2 is expressed in more than $80 \%$ of colorectal cancer. Expression of CK20 combined with CDX-2 can improve the diagnostic accuracy of colorectal cancer [9]. The positive rate of CK19 in gastric cancer is higher than that in colorectal cancer. GCDFP is positive in breast cancer with high specificity but low sensitivity [10].

For patients with supraclavicular lymph node metastasis, a combination of surgical resection and appropriate radiochemotherapy can achieve a good prognosis. According to previous reports, postoperative chemotherapy with FOLFOX regimen can improve the prognosis. The advantages of 18 Fluorine-labeled 2-fluoro-2-deoxyD-glucose positron emission tomography (FDG-PET) in identifying the primary site and determining the correct diagnosis have been proved [11]. A multidisciplinary team is recommended in determining the diagnosis and establishing the treatment strategy for patients with cervical lymph nodes metastases. The development of distant lymph nodes metastases represents advanced disease and therefore, the main purpose of treatment is to improve the quality of life.

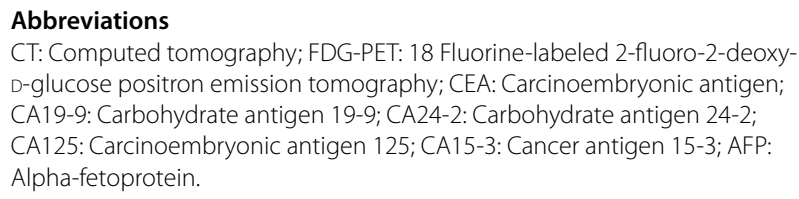

CT: Computed tomography; FDG-PET: 18 Fluorine-labeled 2-fluoro-2-deoxyD-glucose positron emission tomography; CEA: Carcinoembryonic antigen; CA19-9: Carbohydrate antigen 19-9; CA24-2: Carbohydrate antigen 24-2; CA125: Carcinoembryonic antigen 125; CA15-3: Cancer antigen 15-3; AFP: Alpha-fetoprotein.

\section{Acknowledgements}

Not applicable

\section{Authors' contributions}

THX wrote the manuscript. PS and HZ contributed to pathological examination. JGH revised the paper and finally approved manuscript. All authors read and approved the final manuscript.

\section{Funding}

Not applicable.

\section{Availability of data and materials}

Data sharing is not applicable to this article as no datasets were generated or analyzed during the current study.
Ethics approval and consent to participate

Ethics approval by committee was not required for this case report.

\section{Consent to publication}

Written informed consent was obtained from the patient for publication of this case report and any accompanying images.

\section{Competing interests}

The authors declare that they have no competing interests.

Received: 19 October 2020 Accepted: 26 January 2021

Published online: 09 February 2021

\section{References}

1. Nie X, Liu H, Liu L, Wang Y, Chen W. Emerging roles of WNT ligands in human colorectal cancer. Front Oncol. 2020;10:1341.

2. Sun Z, Ma S, Zhou Q, Yang S, Chang Y, Zeng X, Ren W, Han F, Xie X, Zeng F, Sun X, Wang G, Li Z, Zhang Z, Song J, Liu J, Yuan W. Prognostic value of lymph node metastasis in patients with T1-stage colorectal cancer from multiple centers in China. World J Gastroenterol. 2017;23(48):8582-90.

3. Jin M, Frankel W. Lymph node metastasis in colorectal cancer. Surg Oncol Clin N Am. 2018:27(2):401-12.

4. Keum N, Giovannucci E. Global burden of colorectal cancer: emerging trends, risk factors and prevention strategies. Nat Rev Gastroenterol Hepatol. 2019;16(12):713-32.

5. Lopez F, Rodrigo JP, Silver CE, Haigentz M Jr, Bishop JA, Strojan P, Hartl DM, Bradley PJ, Mendenhall WM, Suarez C, Takes RP, Hamoir M, Robbins KT, Shaha AR, Werner JA, Rinaldo A. Cervical lymph node metastases from remote primary tumor sites. Head Neck. 2016;38:E2374-2385.

6. Streu E. Virchow's node and carcinoma of unknown primary. Oncol Nurs Forum. 2015:42(6):688-90.

7. Suliman M, Singh M, Ajmeri A, Stuart D, Teka S. Virchow's node: a case report of an extremely rare presentation of metastasis of adenocarcinoma with mucinous features from the colon. Int J Gen Med. 2019;12:137-40.

8. Fleming M, Ravula S, Tatishchev S, Wang H. Colorectal carcinoma: pathologic aspects. J Gastrointest Oncol. 2012;3(3):153-73.

9. Witek M, Nielsen K, Walters R, Hyslop T, Palazzo J, Schulz S, Waldman S. The putative tumor suppressor $\mathrm{Cdx2}$ is overexpressed by human colorectal adenocarcinomas. Clin Cancer Res. 2005;11:8549-56.

10. Park J, Kim J. Pathologic differential diagnosis of metastatic carcinoma in the liver. Clin Mol Hepatol. 2019;25(1):12-20.

11. Basso L, Izzo L, Calisi E, Cavallaro G, Costi U, Ciardi A, Fornari F, Polistena A, De Toma G. Cervical node metastasis as the first sign of cancer of the caecum. Anticancer Res. 2007;27:3589-92.

\section{Publisher's Note}

Springer Nature remains neutral with regard to jurisdictional claims in published maps and institutional affiliations.

\footnotetext{
Ready to submit your research? Choose BMC and benefit from:

- fast, convenient online submission

- thorough peer review by experienced researchers in your field

- rapid publication on acceptance

- support for research data, including large and complex data types

- gold Open Access which fosters wider collaboration and increased citations

- maximum visibility for your research: over 100M website views per year

At BMC, research is always in progress.

Learn more biomedcentral.com/submissions
} 